\title{
Polar Cap Potential and Merging Electric Field during High Intensity Long Duration Continuous Auroral Activity
}

\author{
Binod Adhikari ${ }^{1}$ and Narayan P. Chapagain ${ }^{2}$ \\ ${ }^{1}$ Department of Physics, Amrit Science Campos, Tribhuvan University, Kathmandu, Nepal \\ ${ }^{2}$ Department of Physics, Patan M. Campus, Tribhuvan University, Patan Gate, Lalitpur. Nepal \\ Corresponding author: Binod Adhikari (binod.adhi@gmail.com)
}

\begin{abstract}
The polar cap potential (PCV) has long been considered as a key parameter for describing the state of the magnetosphere/ionosphere system. The relationship between the solar wind parameters and the PCV is important to understand the coupling process between solar wind-magnetosphere-ionosphere. In this work, we have estimated PCV and merging electric field (Em) during two different high intensity long duration continuous auroral activity (HILDCAA) events. For each event, we examine the solar wind parameters, magnitude of interplanetary magnetic field (IMF), interplanetary electric field (IEF), PCV, Em and geomagnetic indices (i.e., SYM-H, geomagnetic auroral electrojet (AE) index, polar cap index (PCI) and auroral electrojet index lower (AL), respectively). We also study the role of PCI and AL indices to monitor polar cap (PC) activity during HILDCAAs. In order to verify their role, we use wavelet transform and cross-correlation techniques. For the three events studied here, the results obtained from continuous wavelet transform (CWT) and discrete wavelet transform (DWT) are different, however the effect of HILDCAA can be easily identified. We also observe the cross-correlation of PCI and PCV with AL, SYM-H, Bz component of the IMF and Ey component of the IEF individually. Both PCI and PCV show very good correlation with AL and SYM-H indices during the events. Observing these results, it can be suggested that PCI and AL indices play a significant role to monitor geomagnetic activity generated by geoeffective solar wind parameters.
\end{abstract}

Keywords: Magnetosphere, polar cap potential, HILDCAAs, wavelet analysis

\section{Introduction}

The polar cap potential (PCV) has long been considered as an indicator for the development of magnetospheric convection. The relationship between the solar wind parameters and the PCV is important to understand the coupling process between solar wind-magnetosphereionosphere. The connection between the solar wind drivers and convection patterns in Earth's polar ionosphere has been studied extensively for the past 2 decades [Hairston et al., 2005].
Among these solar wind drivers, the role of southward interplanetary magnetic field (IMF) conditions have been understood very well [Richmond and Kamide, 1998; Rich and Hairston, 1994; Boyle et al., 1997; Ruohoniemi and Baker, 1998; Hairston et al., 2005]. As the solar wind embedded with IMF flows toward the Earth, a cross-magnetospheric electric field is generated along the magnetopause. When IMF oriented southward, it reconnects 
directly with the Earth's magnetic field and a part of this electric field transfers to the polar ionosphere. This creates a potential difference in the ionosphere and is known as cross-PCV which can be directly measured from low Earth orbiting satellites [Papitashvili et al., 1999] from radars [Shepherd, 2007] and from ground magnetometers [Kamide et al., 1981].

Several researches have worked in order to understand the relationship between IMF $(\mathrm{Bz})$ and cross-PCV [e.g., Weimer, 1996]. They found that as the magnitude of IMF, Bz increase, the cross-magnetospheric electric field also increases which in turn to increase the ionosphere crossPCV. They also observed that the PCV is a linear function of the solar wind speed and the magnitude of IMF (Bz). The ionospheric and magnetospheric convection state can be obtained through the difference between the maximum and the minimum of the cross-PCV in one hemisphere [Raeder and Lu, 2005].

The saturation of PCV during intense geomagnetic storm has been studied by many researchers [Raeder and Lu, 2005; Shepherd et.al, 2007]. In the language of geophysics saturation means that physical quantities are less than expected for a given strength of solar wind parameters. There are two main reasons for the saturation of PCV. The first one is the decrease in the efficiency of dayside magnetic reconnection at the magnetopause [Hill, 1975]. The other one is the decrease in the polar cap potential induced in the ionosphere [Fedder and Lyon, 1987]. The formal one may be caused by imbalance between the magnetic field intensities whereas the later one may be related to coupling between the magnetosphere and ionosphere. The coupling function plays an important role to understand the relationships between ionosphere-magnetosphere and solar wind energy input [deLucas et al., 2007]. First time, it was derived by Perreault and Akasofu [1978] and showed that interplanetary energy flux is estimated on the basis of the flux of the Poynting vector. Later, it was developed by Kan and Lee [1979] based on the magnetic reconnection geometry. Nagatsuma [2004] studied the saturation of PCV and found that the degree of saturation does not depend on components of IMF but depends on the value of merging electric field.

Similarly, Borovsky et al. [2009] found that the saturation of the PCV occurred statistically during storm-times, solar maximum, and lowMach number solar wind. Troshichev et al. [1996] studied the statistical dependence of the PCV with PCI and showed a linear relationship. The main objective of this work is to study the relationship of polar cap potential and polar cap index with the interplanetary electric field, magnetic field, calculated merging electric field and geomagnetic index AL and SYM-H for three different HILDCAA events. We calculated both PCV and Em as suggested by Kan and Lee [1979]. This work is presented as following. Section 1 describes the datasets and section 2 presents different methodologies use for this work. Section 3 illustrates the results obtained from this analysis and section 4 concludes the results.

\section{Data Set and Methodology}

In the present study, three different HILDCAA events of different interplanetary cases were identified. These events were 
selected using the following four criteria as suggested by Tsurutani and Gonzalez [1987] from the list of HILDCAAs events discussed by Hajra et al. [2013]:

(i) The $\mathrm{AE}$ index is expected to reach over $1000 \mathrm{nT}$ at least once.

(ii) It never occurs below $200 \mathrm{nT}$ for periods longer than two hours at a time.

(iii) These conditions should last at least two days.

(iv) It should occur outside the main phase of geomagnetic storms.

In order to identify HILDCAA events, AE and $\mathrm{AL}$ indices of 1-minute time resolution were used. This dataset is compiled from the OMNI website (lurl\{http://cdaweb.gsfc. nasa.gov/ $\}$ ). The SYM-H indices (1 min time resolution) used to identify the main phase of geomagnetic storm were also obtained from the same source. Interplanetary data sets used in this work are plasma speed (Vsw), density (Nsw), temperature (Tsw) and magnitude of magnetic field $\left(\mathrm{B}_{0}\right)$ and components $(\mathrm{Bx}, \mathrm{By}, \mathrm{Bz})$. Then, Em and PCV were derived as suggested by Kan and Lee [1979] and Moon [2012]. These datasets at $1 \mathrm{~min}$ time resolution were obtained also from the OMNI website. This site gives the high resolution OMNI datasets consisting of 1-min averaged ACE, WIND, IMP-8, Geotail and other solar wind data time-shifted to the bow shock.

In this work, we used wavelet analysis (continuous wavelet transform, discrete wavelet transforms and wavelet scale correlation) and cross-correlation. For continuous wavelet transform, we use here the well known Morlet analyzing function that balances time and scale domain representations [Morlet, 1983; Domingues et al., 2005]. For the algorithm of discrete wavelet transform (DWT), we used Daubechies order 2 orthogonal wavelet transform of 7 levels. Using this technique, we detect the singularities present on polar cap (PC) and AL indices during the HILDCAAs.

\subsection{Continuous Wavelet Transform (CWT)}

A continuous wavelet transform (CWT) is used to divide a continuous-time function into wavelets [Grossmann and Morlet, 1983]. It provides a very redundant and finely detailed description of a signal in terms of both time and frequency. Let ' $a$ ' and ' $b$ ' be the dilation and translation parameters that vary continuously over R. Then the wavelet transform becomes

$$
\mathrm{W}(\mathrm{a}, \mathrm{b})=\int \mathrm{f}(\mathrm{t}) \Psi^{*}((\mathrm{t}-\mathrm{b}) / \mathrm{a}) \mathrm{dt}
$$

Where, * represents the complex conjugate. This function $W(a, b)$ represents the wavelet coefficients. The variations of scale parameter give dilation effect when $a>0$ and contraction effect when $a<0$ of the mother wavelet function. So that it is possible to analyze the low and high frequency or the long and short period features of the signal.

\subsection{Discrete Wavelet Transform (DWT)}

A discrete wavelet transform (DWT) is a wavelet transform in which the wavelets are discretely sampled. It contains discrete values of scale (j) and localization (k). So it may or may not have redundant representation depending on the discretization scheme used [Daubechies, 1992; Domingues et al., 2005]. In discrete wavelet transform the scale and the translation 
parameters give discrete values, i.e., $a=2^{j}$ and $\mathrm{b}=\left(2^{\mathrm{j}}\right) \mathrm{k}$. The discrete wavelet transform can be expressed in terms of wavelet coefficients as

$$
D_{k}^{j}=2^{-j / 2} \int f(t) \Psi^{j-k} d t
$$

where, $\Psi_{k}^{\mathrm{j}}(\mathrm{t})=2^{-\mathrm{j} / 2} \Psi\left(2^{-\mathrm{j}}(\mathrm{t}-\mathrm{k})\right.$. The coefficients $\mathrm{d}_{\mathrm{k}}^{\mathrm{j}}$ are known as "details" (details are higher frequency structures or local approximation errors). For the purposes of our study, the Daubechies orthogonal wavelet of order two is chosen. A more detailed description of the wavelet technique can be found in Domingues et al. [2005]; Mendes et al. [2005]; Ojeda et al. [2014a] and Klausner et al. [2014a].

\section{Results and Discussion}

The polar cap potential has long been considered as a key parameter for describing the state of the magnetosphere-ionosphere system [Boyle et al., 1997], which helps to understand the coupling process between solar wind and this system. In this work, we estimated polar cap potential and merging electric field as suggested by Kan and Lee [1979] during two different HILDCAA events. We examine the interplanetary electric field, merging electric field and polar cap potential, and also study the role of PC and AL indices to monitor polar cap activity during HILDCAAs. The AL index is derived from geomagnetic variation measure from twelve longitudinally spaced stations located at northern auroral latitudes and provides the information about the intensity of westward electrojet in the auroral ionosphere [Ballatore and Maclennan, 1999; Moon, 2012]. On the other hand, the PC index is used for the measurement of current in the polar cap. It is derived from magnetic data at a single groundbased station near-pole [Troshichev, 1988].

\section{Case 1: Non-storm HILDCAA occurred on} April 20-23, 2003

Figure 1 shows an example of non-storm HILDCAA occurred on April 20-23, 2003. The panels are from top to bottom, the solar wind temperature (Tsw in $10^{4} \mathrm{~K}$ ), speed (Vsw in $\mathrm{km} / \mathrm{s}$ ), plasma density (Nsw in $\mathrm{cm}^{-3}$ ), IMF magnitude ( $\mathrm{B}_{0}$ in nT), By (nT), Bz (nT), SYM-H (nT) and AE (nT). The HILDCAA event starts at the mid day of 110 (April 20, 2003) to the beginning of 113 (April 23, 2003). During this time, the components of magnetic field $(\mathrm{Bx}, \mathrm{By}$ and $\mathrm{Bz}$ ) show fluctuation due to the presence of Alfven waves [Guarnieri et al., 2006]. The decrease in SYM-H index is very weak between $-20 \mathrm{nT}$ to $-30 \mathrm{nT}$. The AE index shows intense

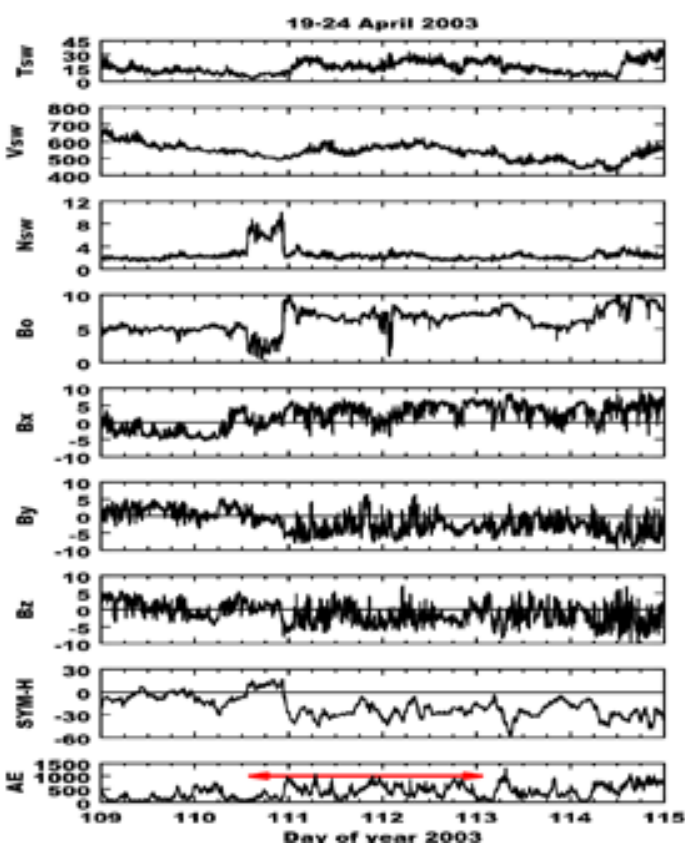

Figure 1. Variations of solar wind temperature (Tsw in $10^{4} \mathrm{~K}$ ), speed (Vsw in $\mathrm{km} / \mathrm{s}$ ), plasma density (Nsw in $\left.\mathrm{cm}^{-3}\right)$, IMF magnitude $\left(\mathrm{B}_{0}\right.$ in $\left.\mathrm{nT}\right), \mathrm{By}(\mathrm{nT}), \mathrm{Bz}$ (nT), SYM-H (nT) and AE (nT). In the figure, the HILDCAA interval is marked by red horizontal arrow in AE panel. It occurs during April 20-23, 2003. 
activity at the mid day of 110 to the beginning of 113. These all above information suggested that the HILDCAA criteria are strictly satisfied as suggested by Tsurutani and Gonzalez [1987].

Figure 2 shows the variation of interplanetary electric field (Ey in $\mathrm{mV} / \mathrm{m}$ ), merging electric field $(\mathrm{Em}$ in $\mathrm{mV} / \mathrm{m})$, polar cap potential $(\mathrm{PCV}$ in $\mathrm{kV})$, polar cap index (PCI in $\mathrm{mV} / \mathrm{m}$ ) and AL (nT) for the HILDCAA event on 20-23 April 2003. The horizontal red line with double arrow on AL index at the last panel shows the HILDCAA interval. During the event, Ey shows significant east-west perturbations. Merging electric field shows

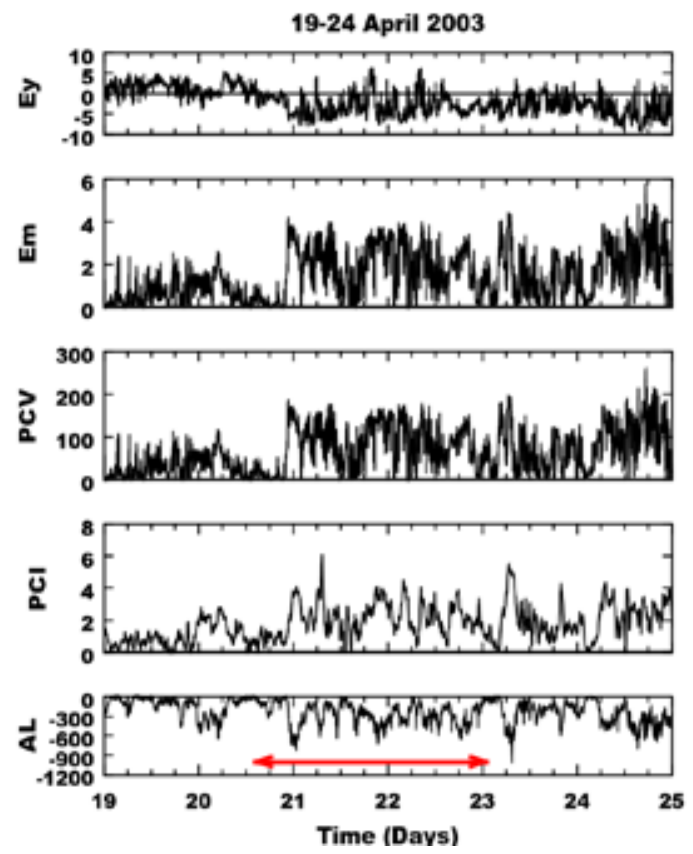

Figure 2. From top to bottom, the panels represent the variations of interplanetary electric field (Ey in $\mathrm{mV} / \mathrm{m})$, merging electric field (Em in $\mathrm{mV} / \mathrm{m}$ ), polar cap potential (PCV in $\mathrm{kV}$ ), and geomagnetic index $\mathrm{PC}(\mathrm{mV} / \mathrm{m})$ and $\mathrm{AL}(\mathrm{nT})$ for the non-storm HILDCAA event. The HILDCAA interval is marked by red horizontal arrow in AL panel. It occurs during 20-23 April 2003. high level of fluctuation through out the event and has an average value of $1.83 \mathrm{mV} / \mathrm{m}$. A more detail about the Em can be found in Vennerstrqm et al. [1991] and Moon [2012]. The polar cap potential $(\mathrm{PCV})$ shows continuous variations during the event and has an average of 81.83 $\mathrm{kV}$. The parameters PCV and Em are derived as suggested by Kan and Lee [1979]. In the figure, the nature of variations on both PCI and AL indices are very well anti correlated, which shows very close relation between them. The HILDCAA time averages for them are -264.98 $\mathrm{nT}$ and $2.08 \mathrm{mV} / \mathrm{m}$ respectively.

In order to verify the role of PCI and AL indices on polar cap potential and merging electric field, we have implemented some wavelet techniques (CWT and DWT) and cross-correlation which are explored below.

Figure 3 shows the scalograms for AL (top) and PCI (bottom) indices for the same event. In the figure, the horizontal axis represents the time in days and the vertical axis represents the scale in minutes. The scale of color on the right side of the second panel has same unit of real data. The scalogram of AL shows different power areas at different times and scales. The power areas of the highest intensity covered by red color are seen at time scales between 150-300 minutes. Similarly, the power areas covered by yellow colors are seen at time scales between 100-300 minutes. The less intense power areas covered by green color are seen at several places. The right side of Figure 3 gives the scalogram for PC index. It shows the strong power areas covered by red color at time scales approximately between 80-300 minutes. In both scalograms, the characteristics of signals 

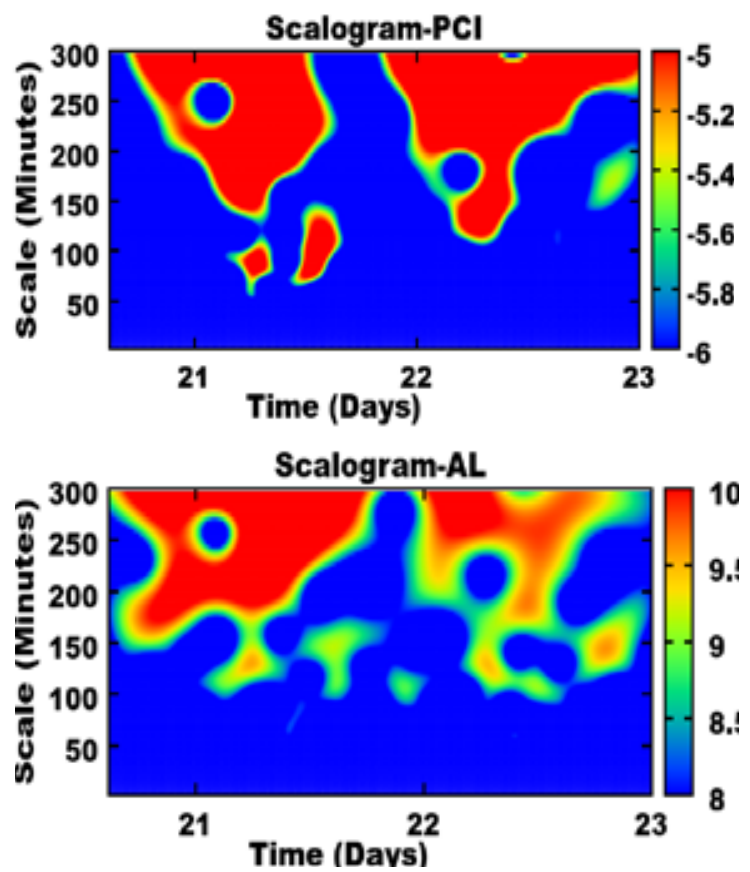

Figure 3. Scalograms for AL (top) and PCI (bottom) during HILDCAA event on April 20-23, 2003.

with highly variable in time with presence of continuous periodicities are presence at higher time scales between 150-300 minutes.

However, the wavelet powers of the highest spectral variabilities are seen at time scales between 10-300 minutes for $\mathrm{AL}$ and 80-300 minutes for PCI. These results show that longer periodicities between 150-300 minutes are most continuous on the series. Observing these results, it can be noticed that some characteristics effects during HILDCAA are seen on both indices. These characteristics show that both $\mathrm{AL}$ and $\mathrm{PC}$ indices were highly disturbed at the time of HILDCAA.

Figure 4 represents the results of discrete wavelet transform for AL (up) and PCI (down) indices for the same event. The Daubechies order 2 orthogonal wavelet transform of seven

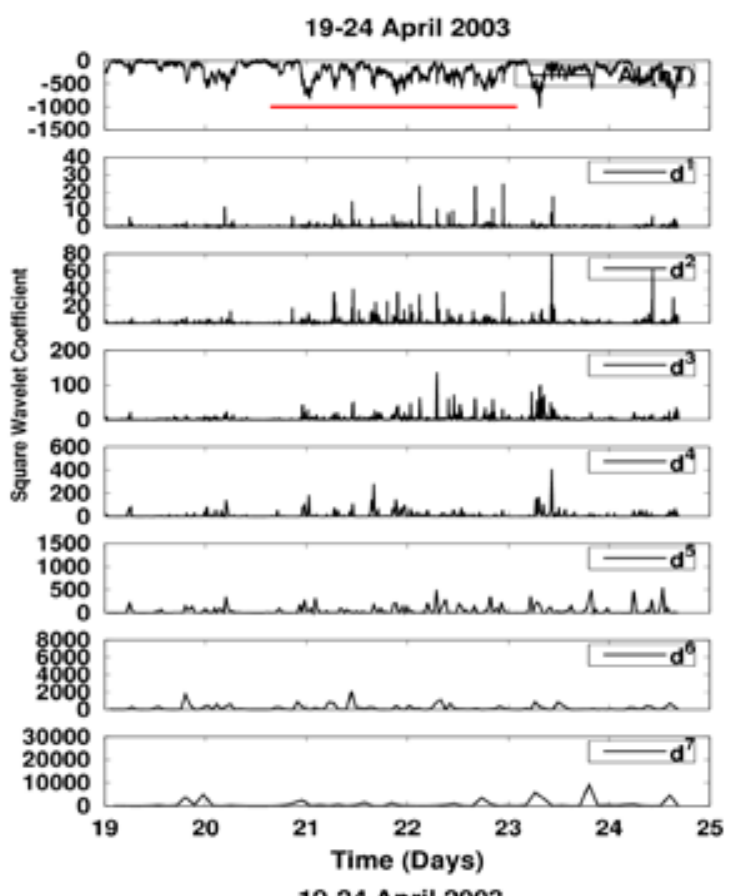

19-24 April 2003

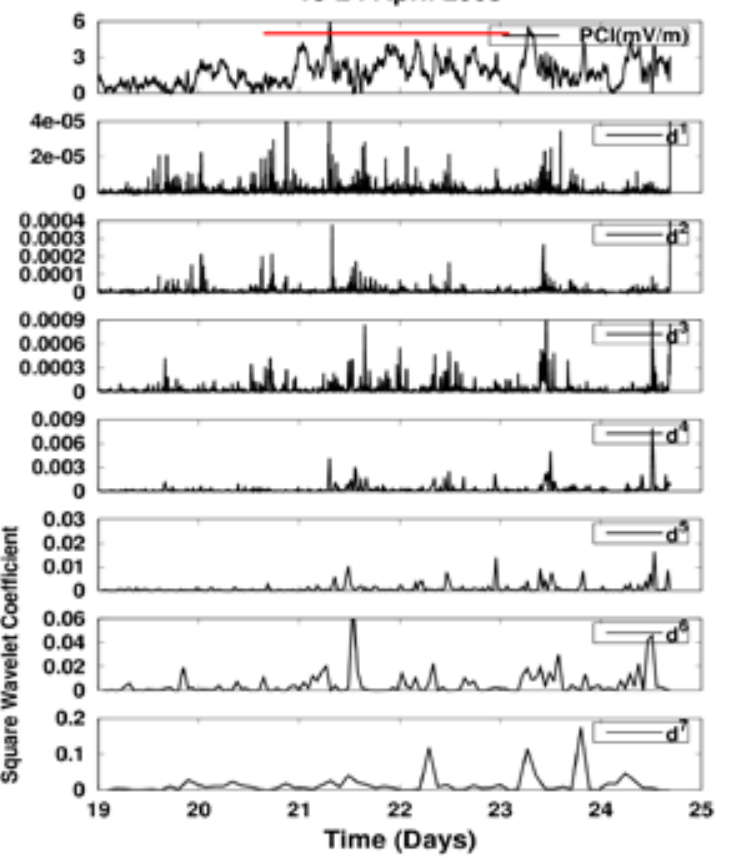

Figure 4. Daubechies Wavelet coefficients $d^{j}$ (for $\mathrm{j}=1,2,3, \ldots .7)$ for AL (Top) and PC (Bottom) indices during HILDCAA event occurred on April 20-23, 2003. The red color identify where the HILDCAA events is happening. 
levels $(\mathrm{j}=1,2,3,4,5,6,7)$ has been used for this analysis. The seven levels are denoted by $\mathrm{d} 1, \mathrm{~d} 2$, d3, d4, d5, d6 and d7. For the chosen wavelet of frequency 0.66667 and sampling rate of one minute, the pseudo periods of the seven levels were 3, 6, 12, 24, 48, 96, 192 minutes. The red marked color in 4 each first panel represents the HILDCAA preceding time. The left side of this figure gives DWT for AL. It shows singularity only at $\mathrm{d} 2$ level. But higher and smaller amplitude of squared wavelet coefficients are present on all levels. However, the amplitude of squared wavelet coefficients for each level is different. Similarly, the right side of this figure gives the result of DWT for PCI. It shows singularities on first four decomposition levels (d1, d2, d3, and d4) at the time of HILDCAA. It also shows singularities after this event, which is related to another HILDCAA event. This index shows relatively larger amplitude of squared wavelet coefficient as compared to $\mathrm{AL}$ index. Observing these amplitude of squared wavelet coefficients, it can be concluded that both indices are highly disturbed and freshly injection of charged particle are carried on at high latitudes during the HILDCAA. A more detail description about the injection of charged particle inside the low, mid and high latitudes of the ionosphere and magnetosphere during geomagnetic disturbances can be found in Morioka et al. [2003].

\section{Case 2: HILDCAA preceded by Co- rotating interaction region-storm occurred on February 12-15, 2004.}

Figure 5 represents the omni datasets for the HILDCAA preceded by Co-rotating interaction region-storm (CIR-storm) occurred on February 12-15, 2004. The data sets from top to bottom represents solar wind temperature
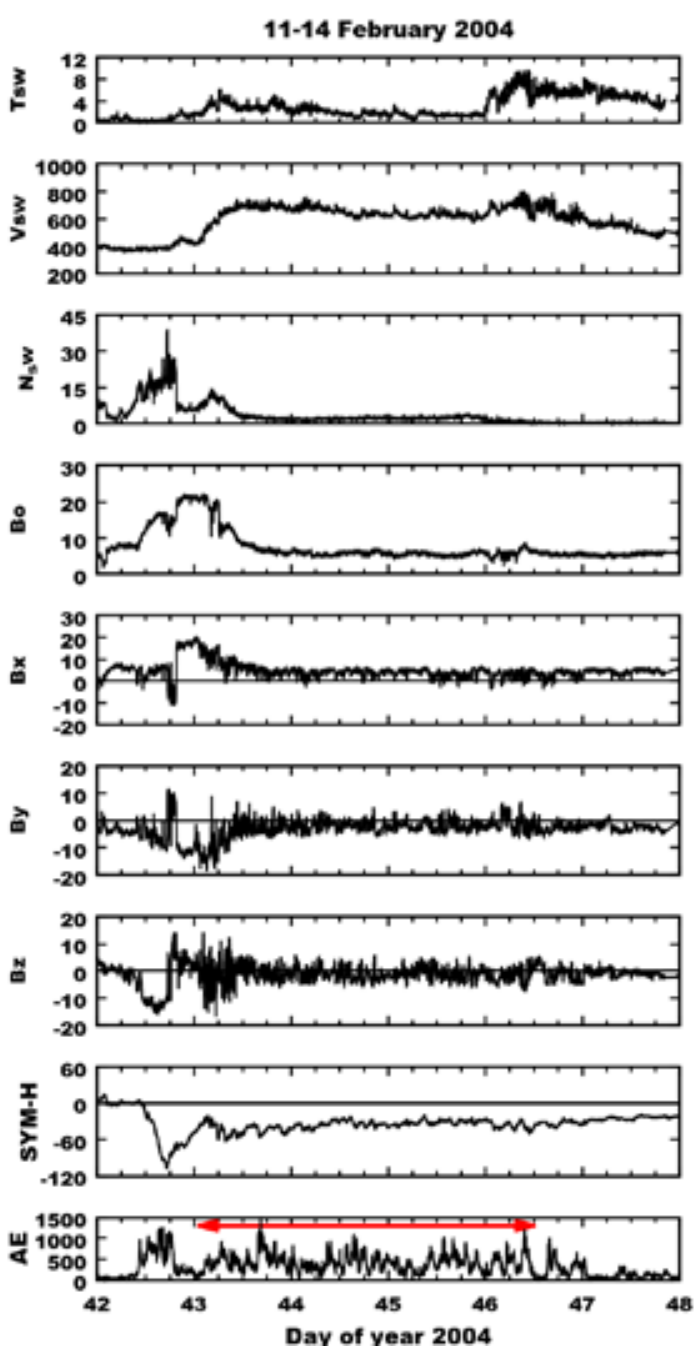

Figure 5. Variations of solar wind temperature (Tsw in $10^{4} \mathrm{~K}$ ), speed (Vsw in $\mathrm{km} / \mathrm{s}$ ), plasma density (Nsw in $\mathrm{cm}^{-3}$ ), IMF magnitude $\left(\mathrm{B}_{0}\right.$ in $\left.\mathrm{nT}\right), \mathrm{By}(\mathrm{nT})$, $\mathrm{Bz}(\mathrm{nT}), \mathrm{SYM}-\mathrm{H}(\mathrm{nT})$ and $\mathrm{AE}(\mathrm{nT})$. In the figure, the HILDCAA interval is marked by red horizontal arrow in AE panel. It occurs during February 11-14, 2004.

(Tsw in $10^{5} \mathrm{~K}$ ), speed (Vsw in $\mathrm{km} / \mathrm{s}$ ), plasma density (Nsw in $\mathrm{cm}^{-3}$ ), IMF magnitude $\left(\mathrm{B}_{0}\right.$ in $\mathrm{nT}), \mathrm{By}(\mathrm{nT}), \mathrm{Bz}(\mathrm{nT}), \mathrm{SYM}-\mathrm{H}(\mathrm{nT})$ and $\mathrm{AE}$ (nT). In this figure, the solar wind parameters and the magnitude of IMF $\left(\mathrm{B}_{0}\right)$ clearly show the Polar Cap Potential and Merging Electric Field 
nature of CIR storm. Initial phase of this storm starts at the late day of 42 (February 11, 2004). The main phase starts around midnight and the long recovery phase (the HILDCAA event) lasts at the beginning of day 43 (February 11, 2004) to mid-day of 46 (February 15, 2004). During this phase, solar wind temperature (Tsw), plasma density (Nsw), speed (Vsw) and magnitude of IMF $\left(\mathrm{B}_{\mathrm{o}}\right)$ show the values of 2 times of $10^{5} \mathrm{~K}, 40 \mathrm{~cm}^{-3}, 400(\mathrm{~km} / \mathrm{s})$ and $20(\mathrm{nT})$, respectively. At the time of HILDCAA, solar wind temperature (Tsw) fluctuated between 2 times $10^{5} \mathrm{~K}$ to 4 times $10^{5} \mathrm{~K}$, speed increases from 400 to $600 \mathrm{~km} / \mathrm{s}$, plasma density (Nsw) and magnitude of $\operatorname{IMF}\left(\mathrm{B}_{0}\right)$ remain almost constant and the IMF components, Bx, By, Bz show high level of fluctuation due to presence of Alfv'en waves [Guarnieri et al., 2006]. The SYM-H index shows strong depression at the main phase and shows almost constant negative value for more than three days at the recovery phase. The AE index at the last panel of this figure shows intense activity and values are always higher for the entire event.

Figure 6 shows the variation of interplanetary electric field (Ey in $\mathrm{mV} / \mathrm{m}$ ), merging electric field ( $\mathrm{Em}$ in $\mathrm{mV} / \mathrm{m}$ ), polar cap potential ( $\mathrm{PCV}$ in $\mathrm{kV}$ ), polar cap index ( $\mathrm{PCI}$ in $\mathrm{mV} / \mathrm{m}$ ) and $\mathrm{AL}(\mathrm{nT})$ for the same event. The horizontal red line with double arrow on $\mathrm{AL}$ index at the last panel shows the HILDCAA event. During the main phase, Ey changes from negative value to positive value and reaches the maximum value being $5 \mathrm{mV} / \mathrm{m}$, the merging electric field was recorded being the maximum value of approximately $7 \mathrm{mV} / \mathrm{m}$, polar cap potential recorded being approximately $250 \mathrm{kV}$, polar cap index had also reached the maximum value recorded as $5 \mathrm{mV} / \mathrm{m}$ and the $\mathrm{AL}$ index reached the minimum value recorded around $-1200 \mathrm{nT}$. But at the time of HILDCAA, the IEF (Ey) shows significant east-west perturbation. The merging electric field also shows strong oscillation and the HILDCAA time average is $1.86 \mathrm{mV} / \mathrm{m}$. The variation in polar cap potential is similar as like merging electric field with an average of $83.32 \mathrm{kV}$. Both PCI and AL indices show intense activity and the HILDCAA time

11-16 February 2004
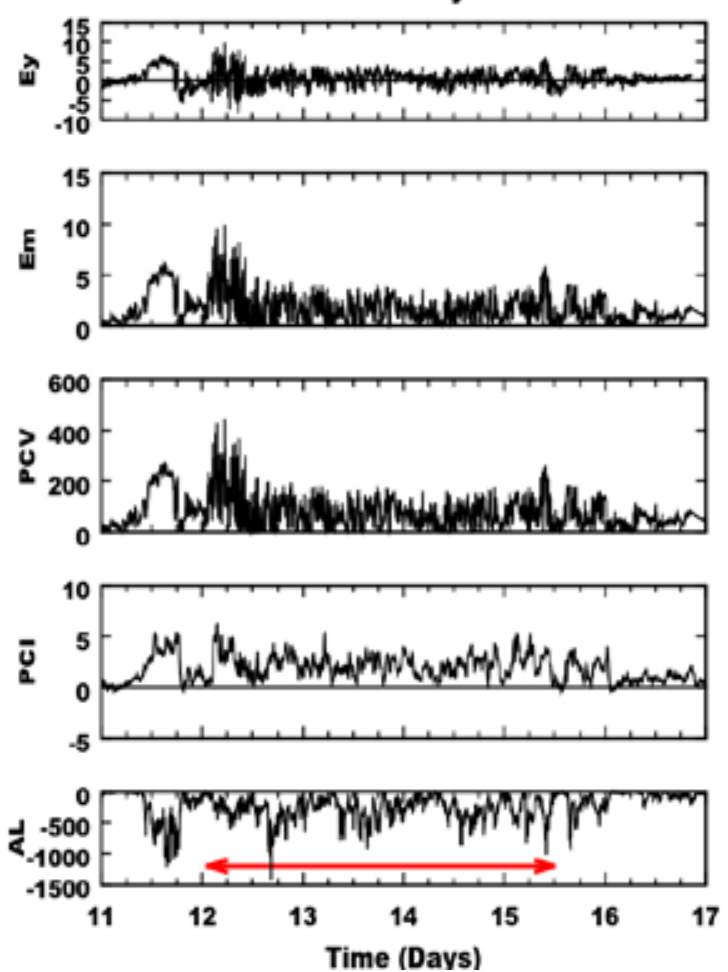

Figure 6. From top to bottom, the panels represent the variations of interplanetary electric field (Ey in $\mathrm{mV} / \mathrm{m})$, merging electric field $(\mathrm{Em}$ in $\mathrm{mV} / \mathrm{m})$, polar cap potential ( $\mathrm{PCV}$ in $\mathrm{kV})$, and geomagnetic index $\mathrm{PC}(\mathrm{mV} / \mathrm{m})$ and $\mathrm{AL}(\mathrm{nT})$ for the non-storm HILDCAA event. The HILDCAA interval is marked by red horizontal arrow in AL panel. It occurs during February 11-14, 2004. 
averages for them are $2.29 \mathrm{mV} / \mathrm{m}$ and -284.94 nT respectively. The HILDCAA time averages value obtained in this event shows slightly greater than previous event for all parameters. It may be related to higher level of Alfvenic fluctuations in IMF-Bz [Guarnieri et al., 2006].

Figure 7 depicts the scalograms for $\mathrm{AL}$ (top) and PCI (down) during HILDCAA event on 12-15 February, 2004. In this event, for both indices, the wavelet powers of the highest spectral variabilities are seen at time scales between 80-300 minutes. As like previous event, the longer periodicities between 200-300 minutes are more continuous on the series.

Figure 8 is similar to Figure 4 but refers to the HILDCAA preceded by CIR-storm occurred on 12-15 February, 2004. As like previous results, the singularities present in these figures also represent the discontinuities
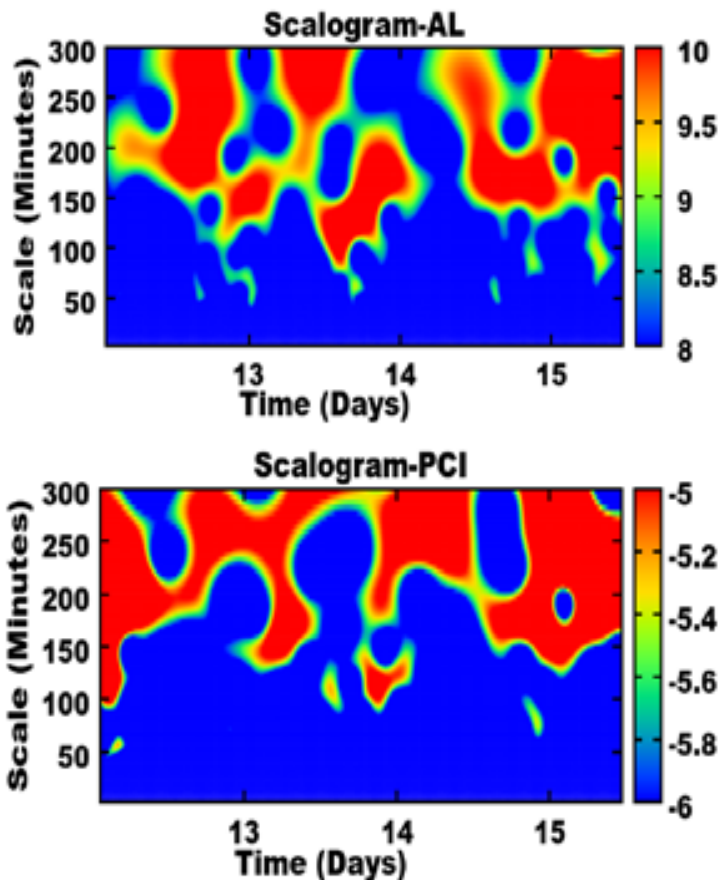

Figure 7. Scalograms for AL (top) and PCI (bottom) during HILDCAA event on February 11-14, 2004.
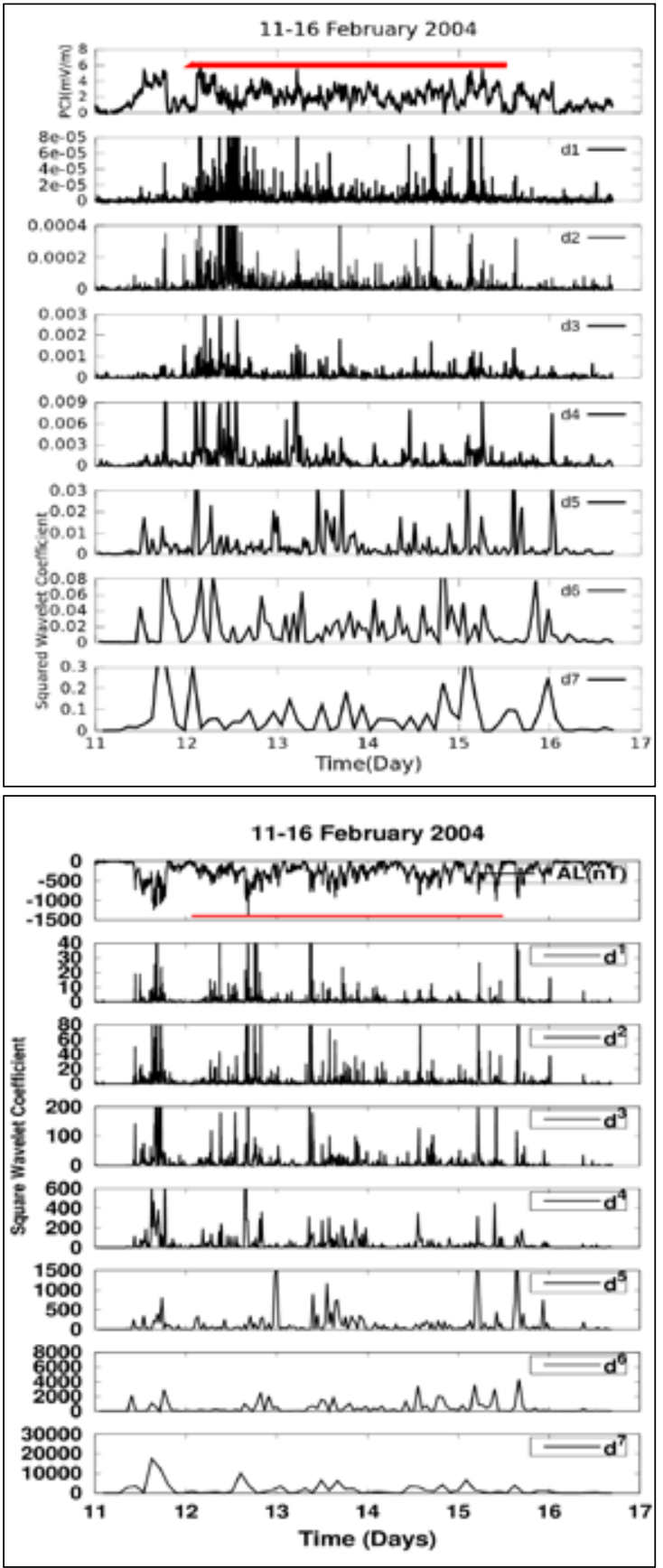

Figure 8. Daubechies wavelet coefficients $d^{j}$ (for $\mathrm{j}=1,2,3, \ldots .7$ ) for AL (top) and PCI (down) indices during HILDCAA event occurred on February 11-14, 2004. The red color identify where the HILDCAA events is happening. 
associated with shocks and higher and smaller amplitude of squared wavelet coefficients represent the energy injection inside the polar cap during the HILDCAA [Mendes et al., 2005; Ojeda et al., 2014b; Klausner et al., 2014b]. Taking into account the amplitude of squared wavelet coefficients, it is found that PCI index shows relatively higher coefficient of squared wavelet coefficients as compared to AL index.

As we know that during the geomagnetic storms, the primary source of the ring current is terrestrial in origin [Hamilton et al., 1988; Sheldon and Hamilton, 1993] and the $\mathrm{O}^{+}$ion is the dominant contributor [Lennartsson and Sharp, 1982; Moore et al., 1999; Lotko, 2007] which shows that magnetospheric processes are capable for energizing low-energy ionospheric ions and transporting them from the ionosphere to the magnetotail and back into the inner magnetosphere. Thus, during HILDCAA, the ionosphere may be the significant sources for the development of ring current.

\section{Conclusions}

The polar cap potential has long been widely considered as an important parameter in characterizing the interaction between solar wind and magnetosphere. However, the measurement of polar cap geomagnetic activity through PCI still is in controversial topic [Moon, 2012]. Although, recently, it has been discussing at the International Association of Geomagnetism and Aeronomy meeting, we have estimated the polar cap potential and merging electric field during two different HILDCAA events. To calculate polar cap potential and merging electric field, we used the relation as suggested by Kan and Lee [1979]. We also studied the role of PC and $\mathrm{AL}$ indices with the polar cap potential and merging electric field during HILDCAAs. In this work, we studied three HILDCAA events having different interplanetary causes and observed solar wind parameters, magnitude of IMF and components, interplanetary electric field, polar cap potential, merging electric field and geomagnetic indices SYM-H, AE, PCI and $\mathrm{AL}$ individually. The results obtained from this work are summarized as below.

For the event occurred on 20-23 April, 2003, the interplanetary electric field shows significant east-west perturbations, while the merging electric field illustrates significant oscillations which has an average value of 1.83 $\mathrm{mV} / \mathrm{m}$. The polar cap potential also shows similar trend as like merging electric field and has an average of $81.83 \mathrm{kV}$. In this event, both $\mathrm{AL}$ and PCI show intense activity and have an average of $-264.98 \mathrm{nT}$ and $2.08 \mathrm{mV} / \mathrm{m}$, respectively. Similarly, for the event on 1215 February, 2004, all these parameters show significant variations as like previous event but the HILDCAA time averages are slightly higher. In conclusion, we have estimated the polar cap potential (PCV) and merging electric field (Em) and studied the role of polar cap (PC) and AL indices during High Intensity Long Duration Continuous Auroral Activity (HILDCAAs), with the major statements:

1. We got significant variations in polar cap potential and merging electric field during the HILDCAAs interval and got different averages for different events.

2. The HILDCAA time averages are higher for ICME preceding HILDCAA and less for non-STORM HILDCAA.

3. All these results suggested that polar cap was severely affected at the time of HILDCAAs. 


\section{Acknowledgments}

Authors thank Dr. Rajkumar Hajra and Dr. Arian. Ojeda González for scientific discussions. Editor N. P. Chapagain thanks the reviewers for their assistance in evaluating this paper.

\section{References}

Ballatore, P., C., Maclennan (1999), Significance of the high-latitude geomagnetic index AES-80 comparison with the PC index, Earth Planets Space, 51, 425-430.

Borovsky, J. E., B. Lavraud, and M. M. Kuznetsova (2009), Polar cap po-tential saturation, dayside reconnection, and changes to the magnetosphere. Geophys. Res., 114, A03224.

Boyle, C. B., P. H Reiff, M .R. Hairston (1997), Empirical polar cap potentials, J. Geophys. Res., 102, 111.

Daubechies, I. (1992), Ten Lectures on Wavelets, CBMS-NSF Regional Conference (Series in Applied Mathematics), Vol. 61. SIAM, Philadelphia, PA.

De Lucas, A., W. D Gonzalez, E. Echer, F.L.Guarnieri, A. Dal Lago, A., M.R da Silva, L.E.A Vieira, N.J.Schuch (2007), Energy balance during intense and super-intense magnetic storms using an Akasofu parameter corrected by the solar wind dynamic pressure. J. Atmos. Solar-Terr. Phys. 69, 1851.

Domingues, M. O., O.J Mendes, A.C.Mendes (2005), On wavelet techniques in atmospheric sciences, Advances in Space Research, 35, pp. 831-842.

Fedder, J. A., and J.G Lyon (1987), The solar windmagnetosphere-ionosphere current-voltage relationship, Geophys. Res. Lett. 14, 880-883.

Grossmann, A. and J. Morlet (1983), Decomposition of Hardy functions into square integrable wavelets of constant shape, SIAM Journal on Mathematical Analysis, 15, 723-73.

Guarnieri, F. F, B. T Tsurutani, W.D. Gonzalez, A.L.C. Gonzalez, M. Grande, F. Soraas, E. Echer (2006), Icme and Cir storms with particular emphases on HILDCAA events, ILWS WORKSHOP.
Hairston, M. R., K.A. Drake, R. Skoug, R. (2005), Saturation of the ionospheric polar cap potential during the October - November 2003 superstorms, J. Geophys. Res., 110, A09S26.

Hajra, R., E. Echer,, B.T. Tsurutani, W.D. Gonzalez, W. D. (2013), Solar cycle dependence of HighIntensity, Long-Duration, Continuous AE Activity (HILDCAA) events, J. Geophys. Res., 118, pp.1-12.

Hamilton, D. C., Gloeckler, G., Ipavich, F. M., Studemann, W., Wilken, B., Kremser, G. (1988), Ring current development during the great geomagnetic storm of February, J. Geophys. Res., 93(A12), 14343-14355.

Hill, T. W. (1975), Magnetic merging in a collisionless plasma, J. Geophys. Res., 80, 4689-4699.

Kamide, Y., A. D., S. Matsushita (1981), Estimation of ionospheric electric fields, ionospheric currents and field-aligned currents from ground magnetic records. J. Geophys. Res. 86, 801-813.

Kan, J.R. and L.C Lee (1979), Energy coupling function and solar wind magnetosphere dynamo, GeoRL, 6, 577 -580.

Klausner, V., G. A. Ojeda, M. O. Domingues, O. Mendes, and A.R.R.Papa (2014a), Study of local regularities in solar wind data and ground magnetograms, J. Atmos. Sol.-Terr. Phy., 112, $10-19$.

Klausner, V., O. Mendes,M. O. Domingues, A.R.R. Papa, R.H.Tyler, P. Frick, E.A. Kherani (2014b), Advantage of wavelet technique to highlight the observed geomagnetic perturbations linked to the Chilean tsunami J. Geophys. Res. Space Physics, 119, 3077-3093.

Lennartsson, O.W., R.D Sharp (1982), A comparison of the $0.1-17 \mathrm{keV} / \mathrm{e}$ ion composition in the near equatorial magnetosphere between quiet and disturbed conditions, J. Geophys. Res. 87 (A8), 6109-6120.

Lotko, W. (2007), The magnetosphere-ionosphere system from the perspective of plasma circulation: A tutorial, J. Atmos. Sol. Terr. Phys., 69, 191-211. 
Mendes, O. J., M.O Domingues, A.C Mendes (2005), Wavelet analysis applied to magnetograms, Journal of Atmospheric and Solar Terrestrial Physics, 67, pp.1827-1836.

Moon, G.H. (2012), Estimation of Polar Cap Potential and the Role of PC Index, Astron. Space Sci. 29(3), 259-267.

Morioka, A., Y. Miyoshi, T. Seki., F. Tsuchiya., H. Misawa, H. Oya, H. Matsumoto., K. Hashimoto, T. Mukai, K. Yumoto, T. Nagatsuma, T. (2003), AKR disappearance during magnetic storms, Journal of Geophysical Research 108 (A6), 1226-1235.

Morlet, J. (1983), Sampling theory and wave propagation, Acoustic Signal/Image Processing and Recognition, (ed C. Chen), in NATO ASI. Springer-Verlag. New York, 1, 233-261.

Nagatsuma T. (2004), Conductivity dependence of cross-polar potential saturation, JGR, 109, A04210.

Ojeda, G. A., O. Mendes., M.A Calzadilla, and M.O Domingues (2013), Spatio-temporal entropy analysis of the magnetic field to help magnetic cloud characterization, J. Geophys. Res., 118, 5403-5414.

Ojeda, G., A., O. Mendes., M.O Domingues., and V.E Menconi (2014a), Daubechies wavelet coefficients: a tool to study interplanetary magnetic fluctuations, Geofis. Int., 53, 101-115.

Ojeda Gonzelez, A., W.D Gonzalez, O. Mendes, M.O Domingues, and R.R Rosa (2014b), Nonlinear fluctuation analysis for a set of 41 magnetic clouds measured by the Advanced Composition Explorer (ACE) spacecraft, Nonlin. Processes Geophys, 21, 1059-1073.

Papitashvili, V.O., F.J Rich. (1999), M.A Heinemann, M.R Hairston, Parameterization of the defense meteorological satellite program ionospheric electrostatic potentials by the interplanetary magnetic field strength and direction. J. Geophys. Res. 104, 177-184.

Perreault, P., S.I Akasofu (1978), A study of geomagnetic storms, GeoJ, 54, 547-573.
Raeder, J., G Lu. (2005), Polar cap potential saturation during large geomagnetic storms, Advances in Space Research 36, 1804-1808.

Rich, F. J., M. Hairston (1994), Large-scale convection patterns observed by DMSP, $J$. Geophys. Res., 99, 3827.

Richmond, A. D., Y. Kamide (1998), Mapping electrodynamic features of the high-latitude ionosphere from localized observations: Technique, J. Geophys. Res., 93, 5741.

Ruohoniemi, J. M., K.B Baker (1998), Largescale imaging of high latitude convection with Super Dual Auroral Radar Network HF radar observation, J. Geophys. Res., 103, 20,797.

Sheldon, R., D. Hamilton (1993), Ion transport and loss in the Earth's quiet ring current, 1. Data and standard model, J. Geophys. Res., 98(A8), 13491-13508.

Shepherd, S.G. (2007), Polar cap potential saturation: Observations, theory, and modeling., Journal of Atmospheric and Solar-Terrestrial Physics 69, 234-248.

Troshichev, O., H. Hayakawa, A. Matsuoka, T. Mukai., K. Tsuruda (1996), Cross polar cap diameter and voltage as a function of PC index and interplanetary quantities, $J G R, 101,13429-$ 13436.

Troshichev, O. A. (1988), The physics and meaing of the existing and proposed high latitude geomagnetic indices, Annales geophysicae, 6(6), 601-610.

Tsurutani, B.T., W.D Gonzalez (1987), The cause of high-intensity long duration continuous AE activity (HILDCAAS): interplanetary alfven wave trains, Planet. Space. Sci., 35, pp. 400-412.

Vennerstrqm, S., E. Friis-Christensen, O.A Troshichev, V.G Andresen (1991), Comparison between the polar cap index PC and the auroral electrojet indicies $\mathrm{AE}, \mathrm{AL}$, and $\mathrm{AU}, J G R$, 96, 101-113.

Weimer, D. R., A flexible, (1996), IMF dependent model of high-latitude electric potentials having space weather applications, Geophys. Res. Lett., 23, 2549. 